Received: March 13, 2009

Returned for revision: April 19, 2009

Accepted after second revision: April 30, 2009

Published online: June 18, 2009

\title{
Biases in Measuring the Brain: The Trouble with the Telencephalon
}

\author{
Lara D. LaDage Timothy C. Roth II Vladimir V. Pravosudov \\ Department of Biology, University of Nevada, Reno, Nev., USA
}

\section{Key Words}

Neuroecology · Telencephalon · Hippocampus •

Food-caching $\cdot$ Chickadees

\begin{abstract}
When correlating behavior with particular brain regions thought responsible for the behavior, a different region of the brain is usually measured as a control region. This technique is often used to relate spatial processes with the hippocampus, while concomitantly controlling for overall brain changes by measuring the remainder of the telencephalon. We have identified two methods in the literature (the HOM and TTM) that estimate the volume of the telencephalon, although the majority of studies are ambiguous regarding the method employed in measuring the telencephalon. Of these two methods, the HOM might produce an artificial correlation between the telencephalon and the hippocampus, and this bias could result in a significant overestimation of the relative hippocampal volume and a significant underestimation of the telencephalon volume, both of which are regularly used in large comparative analyses. We suggest that future studies should avoid this method and all studies should explicitly delineate the procedures used when estimating brain volumes.

Copyright $\odot 2009$ S. Karger AG, Basel
\end{abstract}

\section{Introduction}

It has been suggested that adaptively specialized behaviors correlate with the morphology of particular brain areas. For example, a positive correlation has been reported between caching intensity and the volume of the hippocampus in food-caching birds [Krebs et al., 1989; Sherry et al., 1989; Healy and Krebs, 1996]. Similarly, a positive correlation has been reported between song repertoire size and volume of the song control area (HVC) in songbirds [e.g., DeVoogd et al., 1993; Székely et al., 1996]. To account for overall brain size, a control region of the brain outside of the hippocampus or HVC is also frequently measured. Often times this control region is the remainder of the telencephalon. By controlling for variation in hippocampal/HVC volume due to changes in brain volume outside of changes due to the behavior of interest, we can attempt to attribute differences in these brain regions to particular behaviors. In addition, many large-scale comparative studies also utilize volumetric measures of the brain that might reflect adaptations in behavior, ecology and physiology [e.g., Garamszegi and Eens, 2004; Lucas et al., 2004; Garamszegi and Lucas, 2005; Iwaniuk and Hurd, 2005]. The results from these studies are reliant upon previous studies, such as those from the food-caching paradigm; thus, it is crucial that the data on telencephalon measurements from previous investigations are comparable and accurate.

\section{KARGER}

Fax +41613061234 E-Mail karger@karger.ch www.karger.com (c) 2009 S. Karger AG, Basel

www.karger.com/bbe
Lara D. LaDage

University of Nevada, Reno

Department of Biology, mailstop 314

Reno, NV 89557 (USA)

Tel. +1 (775) 784 4484, Fax +1 (775) 784 1302, E-Mail lladage@unr.edu 
At present, there appear to be two methods to estimate relative hippocampal volume using the volume of the telencephalon. First, telencephalon volume can be calculated in every tissue section in which the hippocampus is present and measured, hereafter referred to as the hippocampal-only method (HOM). In the HOM, the first telencephalic section to be measured is that which also contains the first hippocampal tissue measured. The telencephalon is then measured until the end of the hippocampus. Examples of studies using this technique are Sherry et al. [1993], Cristol et al. [2003], and Day et al. [2005, 2008].

The alternative method of measuring the telencephalon volume is to measure it in its entirety, hereafter in this paper referred to as total telencephalon method (TTM). In the TTM, the telencephalon is measured beginning from the first tissue section of the telencephalon through the last section of the telencephalon. Examples of studies unambiguously using this technique are LaDage et al. [2009] and Roth and Pravosudov [2009]. Most studies, unfortunately, do not explicitly mention which method they use.

This could be a problem, as there appears to be a bias in telencephalon volume estimates when using the HOM. Measuring the telencephalon only in sections in which the hippocampus is present results in an artificial correlation between hippocampal volume and telencephalon volume and a consistent overestimation of relative hippocampal volume. Furthermore, this method also underestimates total telencephalon volume, which would be relevant for large-scale comparative studies that might utilize those data in the future analysis. Also, in relatively small brains, inter-individual variation in the plane of section could have important consequences for estimating telencephalon volume. More importantly, the degree of underestimation of telencephalon volume appears to be dependent on the hippocampal volume and thus produces biases in the measurements of telencephalon volume. We hypothesize that biases in the underestimation of total telencephalon volume will be especially pronounced in brains with smaller hippocampi. Measuring a smaller portion of the telencephalon due to a smaller hippocampus might result in an artificially larger hippocampal-to-telencephalon volume ratio than actually exists; thus, a smaller hippocampus would be represented as relatively larger (when controlling for telencephalon size) than it actually is. This could result in spurious relationships between brain size and ecological variables affecting hippocampal-dependent behaviors such as food-caching. Here we explore this potential bias by com- paring the two different methods of measurement within the same individuals using two food-caching species, black-capped chickadees (Poecile atricapillus) and mountain chickadees (Poecile gambeli).

\section{Materials and Methods}

A subset of data was used from two previous studies in blackcapped chickadees [ $\mathrm{n}=20$; Roth and Pravosudov, 2009] and mountain chickadees [ $n=39$; LaDage et al., 2009]. Because our lab primarily uses these two species, we wanted to see if the potential bias occurred in both species. The black-capped chickadees were captured at five locations in September and October of 2007: Fairbanks, AK (N 64 51' W $\left.147^{\circ} 49^{\prime}\right)$; Prince George, BC (N 53 54' W $122^{\circ} 47^{\prime}$ ); Missoula, MT (N 46 $51^{\prime} \mathrm{W} 114^{\circ} 07^{\prime}$ ); Fort Collins, CO (N $\left.40^{\circ} 39^{\prime} \mathrm{W} 105^{\circ} 11^{\prime}\right)$; and Manhattan, KS (N $39^{\circ}$ $\left.08^{\prime} \mathrm{W} 96^{\circ} 37^{\prime}\right)$. Mountain chickadees were caught near Sagehen Creek in Tahoe National Forest, CA in September of 2007 and January of 2008. All procedures were approved by UNR (A05/0635 and A06/07-25) and UNBC (A2007.0509.015) IACUC and comply with the laws of the USA.

All birds were anesthetized with a lethal overdose of Nembutal $(0.07 \mathrm{ml}$ of $50 \mathrm{mg} / \mathrm{ml} \mathrm{Nembutal})$. The birds were transcardially perfused with $0.1 \mathrm{M}$ phosphate buffered saline for $10 \mathrm{~min}$ followed by $15-20 \mathrm{~min}$ perfusion of $4 \%$ paraformaldehyde in $0.1 \mathrm{M}$ phosphate buffer. Brains were extracted and post-fixed in $4 \%$ paraformaldehyde for 6 days (black-capped chickadees) or 1 day (mountain chickadees) before cryoprotection. Brains were cryoprotected in $15 \%$ sucrose, then $30 \%$ sucrose, and finally flash-frozen on dry ice. Brains were stored at $-80^{\circ} \mathrm{C}$ until sliced. Brains were sliced on a cryostat (Leica CM $3050 \mathrm{~S}:-20^{\circ} \mathrm{C}$ ) in the coronal plane every $40 \mu \mathrm{m}$. Every 4 th section was mounted and Nissl-stained with thionin.

Slides were coded, thus tissue slices were measured blind to treatment. We measured hippocampal volume and the remaining telencephalon volume using standard stereological methods (StereoInvestigator, Microbrightfield, Inc.; microscope, Leica M4000B). Hippocampal volume was measured in its entirety for both methods. For the HOM, we began measurements of the telencephalon with the first tissue section on which we first measured the hippocampus, and we concluded the measurement of the telencephalon on the last measured hippocampus section. For the TTM, telencephalon volume was measured in its entirety. Both methods utilized the Cavalieri procedure [Gundersen and Jensen, 1987] to estimate volume. Hippocampal volume was measured with a $200-\mu \mathrm{m}$ grid; telencephalon volume was measured with a $1,200-\mu \mathrm{m}$ grid following our previous work [Pravosudov et al., 2002; Pravosudov and Omanska, 2005a, b]. Hippocampal and telencephalic volumes were measured on both left and right hemispheres, which were then summed to produce the given values. There were no significant differences between left and right hemispheric measures for either method.

We used a repeated measures ANOVA to compare telencephalon volume estimates between the two methods to determine if using the different methods yielded different results. To detect a difference in hippocampal volume estimations between the two methods, we directly compared the relative hippocampal vol- 


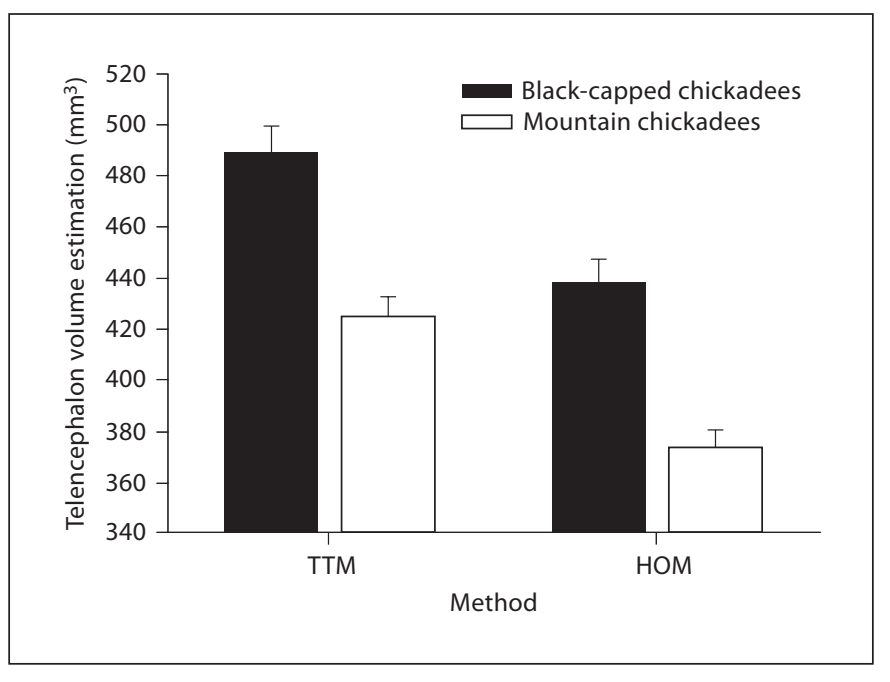

Fig. 1. Estimates using two different methodologies for determining telencephalon volume (HOM = hippocampal only method; TTM $=$ total telencephalon method) in two species of birds. The different methodologies yielded different telencephalon volumes $(\mathrm{p}<0.001)$; telencephalon volumes also differed between species $(\mathrm{p}<0.001)$.

umes (hippocampus volume/telencephalon volume) produced by the HOM and TTM by using repeated measures ANOVA. Furthermore, we tested the effect of the two different methods on the relative hippocampal volume estimation by using the General Linear Model (GLM), with method as a random, independent variable.

To detect potential biases across hippocampal volume estimates, we compared the absolute volume of the telencephalon that is underestimated when using the HOM by subtracting the volume of the telencephalon measured with this procedure from that measured with the TTM. We also compared the proportion of the telencephalon underestimated with the HOM to determine if underestimation of the telencephalon volume was similar across hippocampal volumes. To obtain the proportion of the telencephalon that was underestimated by the HOM, we divided the absolute difference by the volume of the telencephalon when measured with the TTM. We used GLM for all statistical analyses and considered all results to be statistically significant if $\mathrm{p} \leq 0.05$.

\section{Results}

There was a significant difference in telencephalon volumes between the two methods $\left(\mathrm{F}_{1,56}=433.887, \mathrm{p}<\right.$ 0.001 ; fig. 1$)$ and between the two species $\left(\mathrm{F}_{1,56}=31.209\right.$, $\mathrm{p}<0.001)$. We also found a significant difference between relative hippocampal volume estimates when using the TTM compared with the $\operatorname{HOM}\left(\mathrm{F}_{1,56}=358.708, \mathrm{p}<0.001\right.$; fig. 2), but species had no effect $\left(\mathrm{F}_{1,56}=1.686, \mathrm{p}=0.199\right)$.

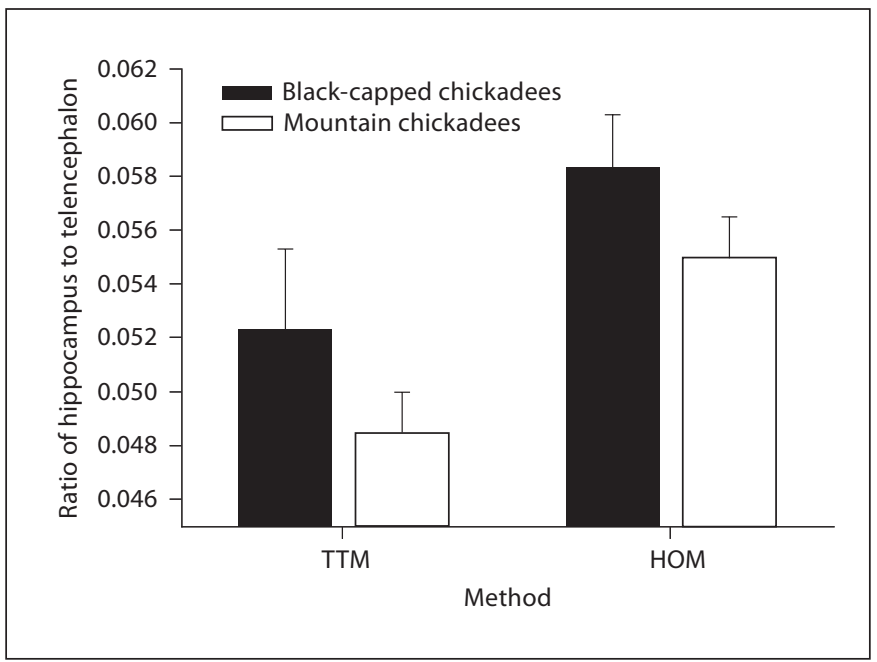

Fig. 2. Estimates of hippocampal volume to telencephalon volume produced by two different methodologies $(\mathrm{HOM}=$ Hippocampal only method; TTM = total telencephalon method) in two species of birds. Hippocampal volume to telencephalon volume differed when using the two different methodologies $(\mathrm{p}<0.001)$, although species had no effect $(\mathrm{p}=0.199)$.

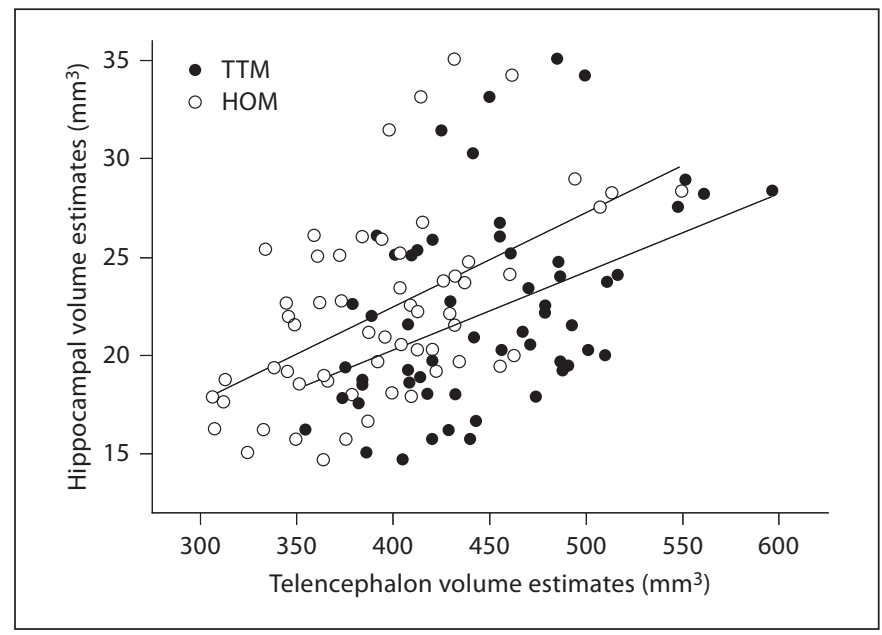

Fig. 3. Relationship between telencephalon volume ad hippocampal volume estimations when measuring the telencephalon with two different methodologies.

Also, the two different methods yielded significantly different relative hippocampal volume estimates when using telencephalon volume estimations as a covariate and method as a random, independent variable $\left(\mathrm{F}_{1,115}=7.11\right.$, $\mathrm{p}=0.008$; fig. 3). 


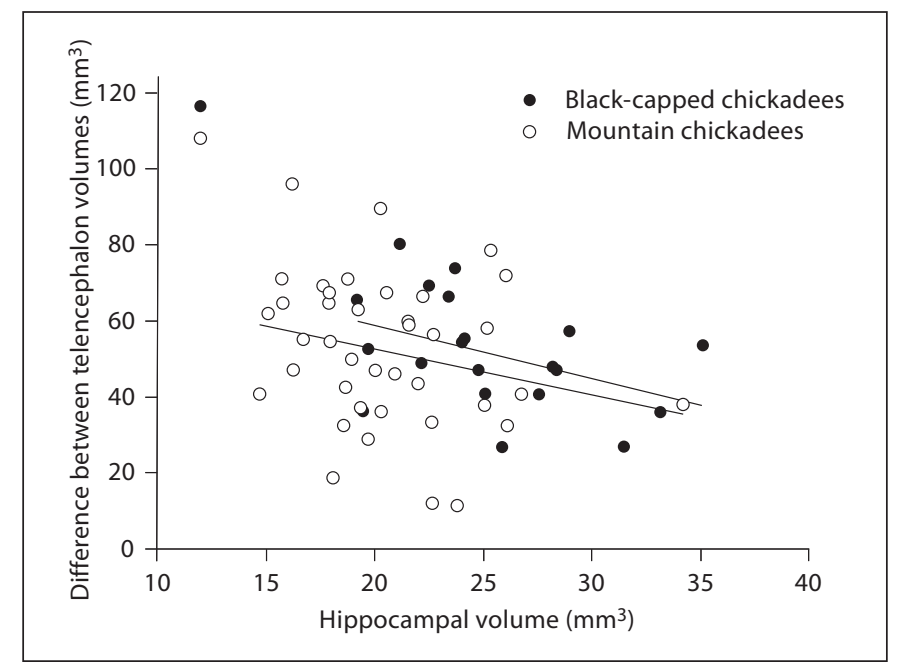

Fig. 4. Relationship between hippocampal volume and the absolute difference in telencephalon volume estimates from two different methodologies.

In addition, we also found a bias across hippocampal volume estimates. There was a significant negative relationship between hippocampal size and the absolute difference between telencephalon volumes estimated using the two methods $\left(\mathrm{F}_{1,56}=5.255, \mathrm{p}=0.026\right)$, whereas species had no effect $\left(\mathrm{F}_{1,56}=1.070, \mathrm{p}=0.305\right.$; fig. 4$)$. We also found a significant negative relationship between hippocampal size and the proportion of the telencephalon that is underestimated when using $\operatorname{HOM}\left(\mathrm{F}_{1,56}=8.140, \mathrm{p}=\right.$ 0.006; again, species had no effect $\left(\mathrm{F}_{1,56}<0.001, \mathrm{p}=0.995\right.$; fig. 5).

\section{Discussion}

The two different techniques produce significantly different telencephalon volume estimations and relative hippocampal volume estimations. The HOM yields telencephalon volume estimations that are significantly lower than those obtained with the TTM (fig. 1), while producing relative hippocampal volumes that are significantly larger than those found with the TTM (fig. 2, 3). Furthermore, using the HOM has differential effects on both the absolute difference in telencephalon volumes estimated using the two methods and the proportion of the telencephalon that is underestimated. The slope of the lines indicate that as hippocampal volume estimates decrease (i.e., measuring a smaller hippocampus), the amount (fig. 4) and the proportion of the telencephalon

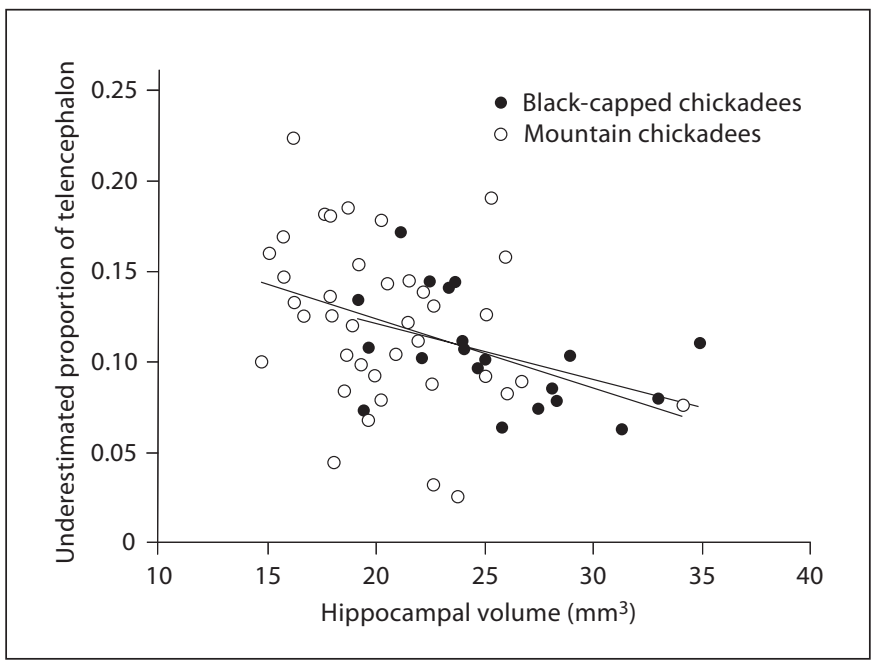

Fig. 5. Relationship between hippocampal volume and the proportion of underestimated telencephalon volume when measuring telencephalon using HOM.

that is underestimated increases (fig. 5). Depending upon hippocampal volume, HOM results in an underestimation of telencephalon volume which ranges from about 5 to $20 \%$ (fig. 5).

Our results indicate that measuring the telencephalon with the HOM might introduce strong biases within a study in which smaller hippocampi are overestimated, thereby masking the true underlying relationship. The direction of this bias indicates that true differences between the species or groups compared could be more difficult to detect when using the HOM. This might not be a problem in studies in which significant differences were detected despite using the HOM, such as in Sherry et al. [1993]. Some studies, however, which have used the HOM did not find differences in relative hippocampal volumes, whereas other studies have found differences; this could be partially attributed to the use of the HOM. For example, Cristol et al. [2003] using the HOM, did not find a significant difference in relative hippocampal volume estimates between migrant and closely-related non-migrant juncos (Junco hyemalis), although both Healy et al. [1996] and Pravosudov et al. [2006] found that migratory species have larger hippocampal volumes relative to the rest of the telencephalon than do sedentary species. Day et al. [2005] used the HOM and did not find a relationship between bower complexity and relative hippocampal volume estimates in bower birds, although, to our knowledge, no other study has examined relative hippocampal volume and bower complexity. Finally, Day et al. [2008] 
also used the HOM and compared relative hippocampal volumes among captive and wild-caught male and female brown-headed cowbirds (Molothrus ater). They found that the only significant difference in relative hippocampal volume was between captive females and wild-caught females. This contrasts with LaDage et al. [2009] in which estimates of relative hippocampal volume using TTM differed between captive and wild-caught male mountain chickadees (Poecile gambeli). Although we do not propose that the use of the HOM alone can mask differences, we do suggest that the use of the TTM could increase the chance of finding differences between relative hippocampal volume estimates, when such differences do exist.

Furthermore, this potential bias might produce a spurious positive correlation between hippocampal volume and telencephalon volume, both within and across species - a relationship that has been proposed as relevant in the literature. For example, food-caching birds have been reported to have larger hippocampal volumes as well as larger telencephalon volumes compared with non-caching species when using brain volume estimates from multiple sources [e.g., Garamszegi and Eens, 2004]. Furthermore, these authors suggest that larger brain regions and overall brain size, similar to the hippocampus, have evolved in response to the demands of food caching. Data from studies which used the HOM were included in the analysis [Sherry et al., 1993; Cristol et al., 2003], thus the telencephalon volumes were likely correlated with the hippocampal volumes in these cases. If a large proportion of the other data were collected using HOM, the reported larger telencephalon volumes found in food-caching birds could be expected simply based on a bias produced by the HOM, a spurious result stemming from the fact that food-caching species have larger hippocampal volumes than non-caching species. Similarly, some withinspecies studies showing a positive correlation between hippocampal and telencephalon volume might also be explained by the biased HOM. Although we cannot, and do not, deny that the positive relationship between hippocampal and telencephalon volumes might exist, we merely suggest that a significant bias resulting from using HOM could be an important factor in some of the reported relationships. This might be particularly problematic in large-scale, comparative, meta-analyses that are unable to define or describe the methodologies used in measuring the telencephalon from the various scrutinized studies. Using biased and underestimated brain sizes in such analyses could lead to spurious conclusions. Consequently, we suggest that such large-scale compara- tive analyses should exercise caution when including data from studies with an unknown methodology.

Although we concede that we do not know the prevalence of the utilization of the HOM, we would like to assume that the majority of studies that do not state their methodology use the TTM. Thus, we view our analysis as a cautionary note for future studies. Clearly, studies investigating relative size of brain areas other than the hippocampus (e.g., song nuclei) might also be affected by similar biases in telencephalon measurements. We suggest that all future studies should utilize the TTM, as it represents the best estimation of volume and circumvents potential biases of measuring telencephalon volume. To avoid ambiguity, we suggest that future studies which correlate behaviors with the morphology of particular brain areas explicitly outline the procedures used in estimating brain volumes, so that other researchers can duplicate the methodology exactly and correctly. This should reduce the likelihood of methodological confusion in future comparative studies as well as potential misinterpretations of brain volumetric patterns as a whole.

\section{Acknowledgments}

We offer our gratitude to many of our colleagues who facilitated our chickadee collection across North America: A. Kitaysky and Z. Kitaiskaia (University of Alaska at Fairbanks); K. Otter (the Otter Laboratory), L. Troc and E. Bayrd (University of Northern British Columbia); C. Breuner and M. Swett (University of Montana at Missoula); P. Doherty, J. Dooley and C. Ghalambor (Colorado State University); and V. Wright, G. Cunningham, E. Horne, B. Sandercock (Konza Prairie Biological Station at Kansas State University). We would also like to thank A. Roth for assistance in the field. Black-capped chickadees were collected under state permits 07-001 (AK), 1603 (MT), 07TRB1100 (CO) and SC009-2007 (KS), and federal permits MB022532-1 (USA), 102990 (USDA import) and 59-07-0198 (Canada). Mountain chickadees were collected under the U.S. Federal Fish and Wildlife (MB022532) and California State (802017-05) scientific collecting permits. This research was supported by grants from the NIH (MH079892 and MH076797) and the NSF (IOB-0615021) to V.V.P. Comments by Sue Healy and an anonymous reviewer significantly improved our manuscript. 


\section{References}

Cristol DA, Reynolds EB, LeClerc JE, Donner AH, Farabaugh CS, Ziegenfus CWS (2003) Migratory dark-eyed juncos, Junco hyemalis, have better spatial memory and denser hippocampal neurons than nonmigratory conspecifics. Anim Behav 66:317-328.

Day LB, Westcott DA, Olster DH (2005) Evolution of bower complexity and cerebellum size in bowerbirds. Brain Behav Evol 66:6272.

- Day LB, Guerra M, Schlinger BA, Rothstein SI (2008) Sex differences in the effects of captivity on hippocampus size in brown-headed cowbirds (Molothrus ater obscurus). Behav Neurosci 122:527-534.

-DeVoogd TJ, Krebs JR, Healy SD, Purvis A (1993) Relations between song repertoire size and the volume of brain nuclei related to song: comparative evolutionary analyses amongst oscine birds. Proc R Soc Lond B 254:75-82.

-Garamazegi LZ, Eens M (2004) The evolution of hippocampus volume and brain size in relation to food hoarding in birds. Ecol Lett 7: $1216-1224$

-Garamazegi LZ, Lucas JR (2005) Continental variation in relative hippocampal volume in birds: the phylogenetic extent of the effect and the potential role of winter temperature. Biol Lett 1:330-333.

-Gundersen HJ, Jensen EB (1987) The efficacy of systematic sampling in stereology and its predictions. J Microsc 147:229-263.
Healy SD, Krebs JR (1996) Food storing and the hippocampus in Paridae. Brain Behav Evol 47:195-199.

Healy SD, Gwinner E, Krebs JR (1996) Hippocampal volume in migratory and non-migratory warblers: effects of age and experience. Behav Brain Res 81:61-68.

Iwaniuk AN, Hurd PL (2005) The evolution of cerebrotypes in birds. Brain Behav Evol 65: 215-230.

Krebs JR, Sherry DF, Healy SD, Perry VH, Vaccarino AL (1989) Hippocampal specialization of food-storing birds. Proc Natl Acad Sci USA 86:1388-1392.

LaDage LD, Roth TC, Fox RA, Pravosudov VV (2009) Effects of captivity and memorybased experiences on the hippocampus of mountain chickadees. Behav Neurosci 123 284-291.

Lucas JR, Brodin A, de Kort SR, Clayton NS (2004) Does hippocampal size correlate with the degree of caching specialization? Proc $\mathrm{R}$ Soc Lond B 271:2423-2429.

- Pravosudov VV, Omanska A (2005a) Prolonged moderate elevation of corticosterone does not affect hippocampal anatomy or cell proliferation rates in mountain chickadees (Poecile gambeli). J Neurobiol 62:82-91.

- Pravosudov VV, Omanska A (2005b) Dominance-related changes in spatial memory are associated with changes in hippocampal cell proliferation rates in mountain chickadees. J Neurobiol 62:31-41.
- Pravosudov VV, Kitaysky AS, Omanska A (2006) The relationship between migratory behaviour, memory and the hippocampus: an intraspecific comparison. Proc R Soc Lond B 273:2641-2649.

- Pravosudov VV, Lavenex P, Clayton NS (2002) Changes in spatial memory mediated by experimental variation in food supply do not affect hippocampal anatomy in mountain chickadees (Poecile gambeli). J Neurobiol 51: 142-148.

Roth TC, Pravosudov VV (2009) Hippocampal volume and neuron numbers increase along a gradient of environmental harshness: a large-scale comparison. Proc R Soc Lond B 276:401-405.

-Sherry DF, Vaccarino AL, Buckenham K, Herz RS (1989) The hippocampal complex of food storing birds. Brain Behav Evol 34:308-317.

-Sherry DF, Forbes MRL, Khurgel M, Ivy GO (1993) Females have a larger hippocampus than males in the brood-parasitic brownheaded cowbird. Proc Natl Acad Sci 90: 7839-7843.

- Székely T, Catchpole CK, DeVoogd A, Marchl Z, DeVoogd TJ (1996) Evolutionary changes in a song control area of the brain (HVC) are associate with evolutionary changes in song repertoire among European warblers (Sylviidae). Proc R Soc Lond B 263:607-610. 


\section{Erratum}

In the article entitled 'Biases in Measuring the Brain: The Trouble with the Telencephalon', by Lara D. LaDage, Timothy C. Roth II, and Vladimir V. Pravosudov, published in Brain Behav Evol 2009;73:253-258, there is a mistake regarding the interpretation of a reference in the discussion. Day et al. 2005 did not use the HOM (hippocampal only method) as stated, instead they measured the telencephalon beginning at the pallialsubpallial lamina and ending on the last section where the hippocampus was discernable, a method that may result in similar problems as the HOM. Additionally, they used body mass, rather than telencephalon, as a correction factor for estimating relative hippocampal volume. 